\title{
Finite Element Modeling of Exotic Options
}

\author{
Jürgen Topper \\ Discussion Paper No. 216 \\ ISSN 0949-9962 \\ Version December 28, 1998 \\ JEL C63, G13 \\ MSC 35K20, 65M60
}

\author{
Jürgen Topper: Arthur Andersen \\ Risikomanagement Beratung \\ Mergenthalerallee 10-12 \\ 65760 Eschborn/Frankfurt \\ Germany \\ Juergen.Topper@De.ArthurAndersen.com
}

Jürgen Topper is a PhD student of Franz Haslinger at the University of Hannover. For their helpful remarks and comments, many thanks to to Dieter Besdo, Kristen Dalton, Hans-Peter Deutsch, Darrel Duffie, Bruno Dupire, Desmond Fitzgerald, Paul Glasserman, Dietmar Hietel, Kenneth Judd, Harold Kushner, Julian Leake, Ulrich Leuchtmann, Israel Nelken, Riccardo Rebonato, Sanjiv Talwar, Marliese Uhrig-Homburg, and Paul Wilmott. 


\section{Abstract}

The Finite Element Method is a well-studied and well-understood method of solving partial differential equations. Its applicability to financial models formulated as PDEs is demonstrated. Its advantage concerning the computation of accurate "Greeks" is delineated. This is demonstrated with various exotic options.

\section{Contents}

$\begin{array}{ll}\text { List of Figures and Tables } & 3\end{array}$

1 Introduction $\quad 4$

2 General Outline $\quad 5$

3 Examples $\quad 7$

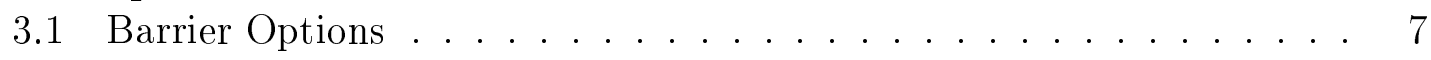

3.1 .1 Double Barrier . . . . . . . . . . . . . 7

3.1 .2 Single Barrier . . . . . . . . . . . . . . . . . 8

3.1.3 Time-dependent Rebates . . . . . . . . . . . . . . . . . 9

3.1.4 Time-dependent Volatilities . . . . . . . . . . . . . 10

3.2 Power Options . . . . . . . . . . . . . . . . . . . . . . . . . . . . . . . . . . . . . .

3.2.1 Plain Vanilla Power Option . . . . . . . . . . . . . . . . 12

3.2 .2 Capped Power Option . . . . . . . . . . . . . 13

3.3 Basket Options . . . . . . . . . . . . . . . . . . . . 15

3.3.1 Put on a Basket . . . . . . . . . . . . . 15

3.3.2 Call on a Basket . . . . . . . . . . . . . . . 18

3.3.3 Single Barrier Knock-Out Call on a Basket . . . . . . . . . . . 20

3.3.4 Double Barrier Knock-Out Call on a Basket . . . . . . . . . . 22

3.3.5 Capped Call on a Basket . . . . . . . . . . . . . . . 23

3.3.6 Capped Power Call on a Basket with a Down-and-out Barrier 24

4 Conclusions $\quad 25$

$\begin{array}{lr}\text { Bibliography } & 26\end{array}$ 


\section{List of Figures}

1 Price of Option as a Function of Time . . . . . . . . . . . . . . 10

2 Premium of a Capped Symmetric Power Option . . . . . . . . . . . . 14

$3 \quad$ Delta $\Delta$ of a Capped Symmetric Power Option . . . . . . . . . . . . . 14

4 Gamma $\Gamma$ of a Capped Symmetric Power Option . . . . . . . . . 15

5 Quadratic and triangular domains . . . . . . . . . . . 17

6 History of FE Mesh . . . . . . . . . . . . . . . . . . . . . . 18

7 Domains of a Single and Double Barrier Knock-out Call . . . . . . . . 21

\section{List of Tables}

1 Data Double Barrier Option . . . . . . . . . . . . . . 7

2 Results Double Barrier Option . . . . . . . . . . . . . . 8

3 Data Single Barrier Option . . . . . . . . . . . . . . . . . . 9

4 Results Single Barrier Option _. . . . . . . . . . . . . . . . 9

$5 \quad$ Results Single Barrier Option with Time-dependent Rebate . . . . . . 10

6 Data Single Barrier Option with Time-dependent Volatility . . . . . . 11

7 Data Volatility Curve . . . . . . . . . . . . . . . . . . . 11

8 Results Volatility Curve . . . . . . . . . . . . . . . 11

9 Data Asymmetric Power Option . . . . . . . . . . . . . . . . . . . 12

10 Results Asymmetric Power Calls (Part 1) . . . . . . . . . . . . . . . 13

11 Results Asymmetric Power Calls (Part 2) . . . . . . . . . . . . . . . . 13

12 Results Capped Asymmetric Power Calls . . . . . . . . . . . . . . . . 14

13 Results Capped Symmetric Power Calls . . . . . . . . . . . . . . . . . 15

14 Data Put on a Basket . . . . . . . . . . . . . . . . . 16

15 Results Put Option on a Basket Computed on a Square Domain . . . 17

16 Results Put Option on a Basket Computed on a Triangular Domain . 18

17 Data Call on a Basket . . . . . . . . . . . . . . . . 19

18 Rubinstein's example with 100 time steps . . . . . . . . . . . . . . 19

19 Rubinstein's example with Dirichlet Boundary Conditions . . . . . . 20

20 Rubinstein's example with Neumann Boundary Conditions . . . . . . 20

21 Results Knock-out Call on a Basket without Rebate . . . . . . . . . . 22

22 Results Knock-out Call on a Basket with Rebate . . . . . . . . . . . . 22

23 Results Double Barrier Knock-out Call on a Basket . . . . . . . . . . 23

24 Results Knock-out Call on a Basket with Rebate . . . . . . . . . . . . 23

25 Results Capped Call on a Basket with Analytical Boundary Conditions 24

26 Results Capped Call on a Basket with Numerical Boundary Conditions 24

27 Results Power Call on a Basket with Floor and Knock-out Barrier . . 25 


\section{Introduction}

Many pricing models can be cast into continuous time and as a result will naturally lead to partial differential equations. These types of PDEs are usually linear and parabolic. In order to avoid clutter in notation we restrict our attention to the case of linear models depending on maximal two factors. ${ }^{1}$ These models have been solved traditionally with Finite Differences (FD). Many different FD techniques exist ([1], ch. 2); the most important have been introduced to financial problems ([28], ch. 15; [14], ch. 10; [50], ch. 16-22; [15]). The usefulness of Finite Elements (FE) has been recognized by many authors ([24], p. 47; [14], p. 212; [16], p. 1664; [17], p. 582; [18], p. 586; [46]; [9], [55], sec. 2.5.4) but to our knowledge the first to explore this idea in some more detail were [31], [32], [21], [22], [23], and [48].

These authors have shown that FE approaches offer some advantages:

- A solution for the entire domain is computed, instead of isolated nodes as in the case with FD codes.

- The boundary conditions involving derivatives are difficult to handle with FD ([20], p. 501). Neumann conditions, however, are often easier to obtain than Dirichlet conditions when estimating the behaviour of the option when the price of the underlying goes to infinity. FE techniques can incorporate boundary conditions involving derivatives easily.

- In addition, FE can easily deal with high curvature. In most FE codes this is achieved by adaptive remeshing, a technique well-developed in theory and in practice.

In this paper we will concentrate on some further advantages of FE:

- The irregular shapes of the PDE's domain can easily be handled while in a FD setting, the placing of the gridpoints is difficult. These irregular domains arise naturally when knock-out barriers are imposed on a multiple-asset option. Irregular shapes can also arise when only parts of the PDE's domain are to be approximated numerically because some parts can be determined by financial reasoning.

- Most academic papers are concernened with pricing only while most practioners are at least as much interested in measures of sensitivity to those prices. Some of these measures of sensitivity, commonly called Greeks, can be obtained more exactly with FE.

- Many FE codes (such as PDEase2D $D^{T M}$ used for this paper) allow local refinement. This allows precise local information without having to solve the problem with accuracy on the entire domain. $P D E$ ase $2 D^{T M}$ also employs adaptive remeshing. This feature automatically leads to local refinement in

\footnotetext{
${ }^{1}$ Most PDE-based option and bond pricing models belong to this class of problem. Notable exceptions are the nonlinear models with transaction cost ([37]; [50], ch. 13; [51]) and the 3-factor swaption model by Dempster and Hutton ([12]; [13]). These models can also be solved with FE, but this will not be demonstrated here; see, for instance [54].
} 
areas of high curvature, for example near to the strike price or close to the barrier.

We will demonstrate these ideas with options which are currently traded in the marketplace. Some of them are listed on stock exchanges. While some of these products are a simple application of the FE approach, many are more sophisticated. We present an approach for valueing options on baskets with various barrier features. This implies a two step procedure: First, some PDEs have to be solved in order to get boundary conditions, and second, another PDE has to be approximated numerically to price the product.

\section{General Outline}

The Pricing PDE Our aim is to explain some features from FE modeling which are especially useful for option pricing. As in most codes available today this takes place within a hybrid $F D / F E^{2}$ framework. This method discretizes time with FD and the spatial variables with $\mathrm{FE}$, and has been, until today, the predominant way of dealing with time in FE analysis. Technical derivations with increasing levels of rigor can be found in [8], [4], [1], and [45]. We convert the original backward parabolic problem into a forward parabolic problem to be in accordance with most numerical literature. The interpretation of $\tau=T-t$ therefore is time to maturity so that the task is to an approximate solution to the following problem: ${ }^{3}$

$$
\begin{aligned}
\frac{1}{2} \sigma_{1}^{2} S_{1}^{2} \frac{\partial^{2} f}{\partial S_{1}^{2}}+\frac{1}{2} \sigma_{2}^{2} S_{2}^{2} \frac{\partial^{2} f}{\partial S_{2}^{2}} & +\rho \sigma_{1} \sigma_{2} S_{1} S_{2} \frac{\partial^{2} f}{\partial S_{1} \partial S_{2}}+ \\
\left(r-q_{1}\right) S_{1} \frac{\partial f}{\partial S_{1}}+\left(r-q_{2}\right) S_{2} \frac{\partial f}{\partial S_{2}} & =r f+\frac{\partial f}{\partial \tau} \\
f\left(S_{1}, S_{2}, 0\right) & =g_{1}\left(S_{1}, S_{2}\right) \text { in } D \\
f\left(S_{1}, S_{2}, \tau\right) & =g_{2}\left(S_{1}, S_{2}, \tau\right) \text { on } R_{1} \\
\frac{\partial f}{\partial n} & =g_{3}\left(S_{1}, S_{2}, \tau\right) \text { on } R_{2} \\
R_{1} \cup R_{2} & =R
\end{aligned}
$$

$D$ is the interior of the convex domain, and $R$ constitutes the boundary. $\frac{\partial f}{\partial n}$ denotes the gradient perpendicular to the boundary. Although boundary conditions eq. (3) and eq. (4) and initial conditon eq. (2) may not be compatible, the problem is wellposed [50]. The equations above can be used to price European options of many kinds as the examples in the following chapter will show. We employ a two-asset formulation of the Black-Scholes equation because the extension to more dimensions is fairly straightforward from a financial and a numerical point of view, since this approach incorporates correlations between the assets and allows for Finite Elements with different geometric shapes. A general FE solution for European and American options has been delivered by [23]. The pricing of American options, however, is more

\footnotetext{
${ }^{2}$ This term stems from Darrell Duffie; the typical name for this approach in the mathematical and engineering literature is time-dependent FE methods.

${ }^{3}$ All notation is based on [28] with the only exception of $S_{n}$ denoting the price of the $n$th underlying (instead of the price on the underlying $S$ at time $n$.)
} 
difficult because an early exercise has to be taken into account. In the PDE setting this naturally leads to moving boundary problems [50] which can also be solved with FE [11]. In the option pricing setting there are currently two approaches: Either the nodes in the elements are manipulated in the same way as they are in a FD setting ([22], p. $7 \mathrm{f})$, or the problem is reformulated as a variational inequality which is solved with FE ([50], p. 410ff).

The problem stated above is a special case of the convection-diffusion problem which has been studied for many decades. Therefore, many numerical techniques are available. One of these is the FEM which is outlined in many textbooks; see for instance [4], [8], and [52]. Here, we do not want to add another general outline of an FE procedure for parabolic problems. Instead, we want to highlight some features which are useful for option pricing in a readable manner. For European options as stated in eq. (1) to (5) the hybrid FD/FE method leads to the following system of ordinary differential equations.

$$
\begin{aligned}
\boldsymbol{q} & =\boldsymbol{A} \boldsymbol{u}+\boldsymbol{B} \dot{\boldsymbol{u}} \\
\boldsymbol{u}(0) & =\boldsymbol{u}_{\mathrm{o}}
\end{aligned}
$$

Thus the problem of solving a PDE has been reduced to solving a system of ordinary differential equations. This initial value problem is usually solved with a FD technique. For a discussion of the most appropriate ones in this setting compare [4] or [8]. The assembly of the elements has been performed implicitly.

Adaptive Time Steps Our software uses Crank-Nicholson time differencing to solve the system above. In order to get an estimate of the error incurred by the time steps a three-step approach is used. First, a half-step solution estimates the values at the mid-step; then, a full-step estimates the values at step end. Then a half-step advances from mid-step to end-step. These two estimates of the end-step value allow the determination of a cubic time term in a Taylor expansion of the solution in time. This cubic term in under the control of the user (by the command errlim).

Adaptive Meshing Since $P D E$ ase $2 D^{T M}$ controls adaptively timesteps and spatial gridding there is a problem of dividing the errors between temporal and spatial controls. The technique employed here is propriatory. So, we will concentrate on the spatial meshing. The software uses triangular elements. ${ }^{4}$ This allows to discretrize any domain with piecewise linear boundaries. Curved boundaries can only be dicretized approximately but this is no disadvantage for financial applications where all the boundaries are linear. In areas of high curvature the triangular elements are divided into two new triangular elements. This process is repeated until some error limit is met.

The Greeks Besides option premiums, one is also interested in the Greeks. The FEM is especially for Delta $\left(\Delta=\frac{\partial f}{\partial S_{i}}\right)$ and $\operatorname{Gamma}\left(\Gamma=\frac{\partial^{2} f}{\partial S_{j} \partial S_{i}}\right)$, well-suited because

\footnotetext{
${ }^{4} P D E$ ase $2 D^{T M}$ treats problems with only one spatial variable as having two spatial variables. The second variable is a dummy.
} 
it delivers a polynomial approximation in the spatial variables. ${ }^{5}$ The derivatives of polynomials can be easily computed analytically and as a result very fast. ${ }^{6}$ Obviously, for this to work, the shape functions have to be at least quadratic. For higher Greeks, like Speed, $\left(\frac{\partial^{3} f}{\partial S_{i} \partial S_{j} \partial S_{k}}\right.$; compare $([55]$, p. 78$)$ this approach becomes complicated due to the fact that many types of elements become admissible. One can improve this procedure by taking the Greeks at the so-called Moan Points. Moan points are points of the FE approximation which have exact derivatives ([42], [4], [8]). Since in financial problems, one is usually interested only in solutions to one or several points in the parameter space, the elements can always be constructed in a way that these points of interest become Moan points. Another possible approach is to use low-order shape functions and employ a device called local smoothing from the engineering literature [27].

\section{Examples}

\subsection{Barrier Options}

\subsubsection{Double Barrier}

We consider an up-and-out-down-and-out call option continuously monitored, ${ }^{7}$ with the following data:

\begin{tabular}{|l|c|}
\hline Parameter & Value \\
\hline \hline Strike price & 100 \\
\hline Down-and-out barrier & 75 \\
\hline Up-and-out barrier & 130 \\
\hline Rebates & none \\
\hline Interest rate & 0.1 \\
\hline Volatility & 0.2 \\
\hline Maturity & 1 year \\
\hline
\end{tabular}

Table 1: Data Double Barrier Option

This leads to the following well-posed backward parabolic PDE problem:

$$
\begin{aligned}
\frac{\partial f}{\partial t}+r S \frac{\partial f}{\partial S}+\frac{1}{2} \sigma^{2} S^{2} \frac{\partial^{2} f}{\partial S^{2}} & =r f \\
f(T, S) & =\max (S-X, 0) \\
f(t, 75) & =0 \\
f(t, 130) & =0
\end{aligned}
$$

The pricing PDE eq. (8) is the famous Black-Scholes equation [5]. Eq. (9) constitutes the payoff at maturity. Eq. (10) and (11) are the knock-out barriers. The analytical

\footnotetext{
${ }^{5}$ There are pure FE approaches which apply FE in time, too; compare [34], [19]. This, however, is not the general methodology.

${ }^{6}$ The package used for this paper [38] only allows numerical derivatives.

${ }^{7}$ Solutions to problems with discrete monitoring can be found by applying the adjustment formulae by [7] to the continuous-monitoring solution.
} 
solution involves a series which goes from $-\infty$ to $\infty$ ([26], p. 73). For numerical purposes this series has to be cut off after some finite number of terms. It has been shown in [35] that it is sufficient to consider only the terms from -2 to 2 because all other terms are very close to zero. Here, for the analytical solution, we have taken the terms from -5 to 5.8 The root mean sqare error RMS is controlled by the user. The default, which is used for all other runs, is 0.001 ([38], p. 104). The value of the underlying is varied in order to catch different degrees of the moneyness. Since the program is adaptive in time and space the number of cycles, nodes, and cells are chosen during the solution process by the program. The code to this and all the other problems from this paper can be found on the disk which comes with this paper. $^{9}$

\begin{tabular}{|c|c|c|c|c|c|c|c|}
\hline \multirow[t]{4}{*}{ Underlying } & \multicolumn{7}{|c|}{ Fair Value } \\
\hline & \multirow[t]{3}{*}{ Analytical } & \multicolumn{6}{|c|}{ Numerical } \\
\hline & & \multicolumn{4}{|c|}{ RMS 0.001} & \multicolumn{2}{|c|}{ RMS 0.0001} \\
\hline & & & Error & & Error & & Error \\
\hline 76 & 0.27306 & 0.27376 & $0.26 \%$ & 0.27317 & $0.04 \%$ & 0.27317 & $0.04 \%$ \\
\hline 80 & 1.22027 & 1.22357 & $0.27 \%$ & 1.22092 & $0.05 \%$ & 1.22087 & $0.05 \%$ \\
\hline 90 & 2.90287 & 2.90875 & $0.20 \%$ & 2.90378 & $0.03 \%$ & 2.90378 & $0.03 \%$ \\
\hline 100 & 3.52511 & 3.52456 & $0.02 \%$ & 3.52395 & $0.03 \%$ & 3.52533 & $0.01 \%$ \\
\hline 110 & 2.89967 & 2.89187 & $0.27 \%$ & 2.89670 & $0.10 \%$ & 2.89932 & $0.01 \%$ \\
\hline 120 & 1.47489 & 1.46833 & $0.44 \%$ & 1.47269 & $0.15 \%$ & 1.47458 & $0.02 \%$ \\
\hline 129 & 0.13192 & 0.13137 & $0.42 \%$ & 0.13181 & $0.08 \%$ & 0.13192 & $0.01 \%$ \\
\hline \multicolumn{8}{|c|}{ Data of FE-Run } \\
\hline \multicolumn{2}{|c|}{ Cycles } & \multicolumn{2}{|c|}{25} & \multicolumn{2}{|c|}{57} & \multicolumn{2}{|c|}{72} \\
\hline \multirow{2}{*}{\multicolumn{2}{|c|}{ Nodes }} & \multirow{2}{*}{\multicolumn{2}{|c|}{$\frac{223}{74}$}} & \multicolumn{2}{|c|}{219} & \multirow{2}{*}{\multicolumn{2}{|c|}{219}} \\
\hline & Cells & & & \multicolumn{2}{|c|}{72} & & 130 \\
\hline
\end{tabular}

Table 2: Results Double Barrier Option

The root mean sqare error RMS is controlled by the user. Error is defined as relative deviation:

$$
\text { error }=\left|\frac{\text { result }- \text { result }_{\mathrm{FE}}}{\text { result }}\right|
$$

The reported errors and differences here and in following sections are based on more significant digits than are shown in the tables.

\subsubsection{Single Barrier}

The following example is based on the example in ([3], p. 225f).

\footnotetext{
${ }^{8}$ It is the normal case that analytical solutions to option pricing problems involve infinite series and/or indefinite integrals. This has led ([50], p. 261) to the recommendation not to look for analytical solutions (which are usually not easy to find provided they exist; compare ([43], sec. 2.3)) but to solve the PDE with numerical methods directly.

${ }^{9}$ Available from the author upon request.
} 


\begin{tabular}{|l|c|}
\hline Parameter & Value \\
\hline \hline Strike price & 100 \\
\hline Up-and-out barrier & 110 \\
\hline Rebate & 10 \\
\hline Interest rate & 0.05 \\
\hline Volatility & 0.2 \\
\hline Maturity & 0.5 year \\
\hline
\end{tabular}

Table 3: Data Single Barrier Option

This leads to the following well-posed backward parabolic PDE problem:

$$
\begin{aligned}
\frac{\partial f}{\partial t}+r S \frac{\partial f}{\partial S}+\frac{1}{2} \sigma^{2} S^{2} \frac{\partial^{2} f}{\partial S^{2}} & =r f \\
f(T, S) & =\max (S-X, 0) \\
f(t, 0) & =0 \\
f(t, 110) & =10
\end{aligned}
$$

Eq. (15) can be interpreted as a knock-out barrier: Once the price of the underlying equity hits zero the company is bankrupt and will not recover in value [33]. Consequently, any call on this equity will be worthless. In eq. (16) a lump sum rebate is introduced.

\begin{tabular}{|c|c|l|l|l|}
\hline Und. & Method & Fair value & Delta & Gamma \\
\hline \hline 80 & Analytical & 0.43223 & 0.08507 & 0.01295 \\
\hline & Numerical & 0.43221 & 0.08507 & 0.01298 \\
\hline & Error & $0.0040 \%$ & $0.0000 \%$ & $0.1965 \%$ \\
\hline \hline 90 & Analytical & 2.10253 & 0.26128 & 0.01999 \\
\hline & Numerical & 2.10252 & 0.26130 & 0.01992 \\
\hline & Error & $0.0003 \%$ & $0.0068 \%$ & $0.3707 \%$ \\
\hline \hline 100 & Analytical & 5.60968 & 0.42205 & 0.00939 \\
\hline & Numerical & 5.60975 & 0.42204 & 0.00927 \\
\hline & Error & $0.0012 \%$ & $0.0014 \%$ & $1.3159 \%$ \\
\hline \hline 105 & Analytical & 7.79972 & 0.44635 & 0.00031 \\
\hline & Numerical & 7.79971 & 0.44635 & 0.00030 \\
\hline & Error & $0.0001 \%$ & $0.0000 \%$ & $3.3333 \%$ \\
\hline \hline 109 & Analytical & 9.56930 & 0.43406 & -0.00625 \\
\hline & Numerical & 9.56929 & 0.43405 & -0.00620 \\
\hline & Error & $0.0001 \%$ & $0.0029 \%$ & $0.8342 \%$ \\
\hline
\end{tabular}

Table 4: Results Single Barrier Option

\subsubsection{Time-dependent Rebates}

We consider the same problem as in sec. (3.1.2) except that the rebate becomes a step-like function of time. Each month it doubles, starting with 1. In mathematical terms, eq. (16) has to be replaced by: 


$$
f(t, 110)=\left\{\begin{array}{cl}
1, & 0<t<\frac{1}{12} \\
2, & \frac{1}{12}<t<\frac{2}{12} \\
4, & \frac{2}{12}<t<\frac{3}{12} \\
8, & \frac{3}{12}<t<\frac{4}{12} \\
16, & \frac{4}{12}<t<\frac{5}{12} \\
32, & \frac{5}{12}<t<\frac{6}{12}
\end{array}\right.
$$

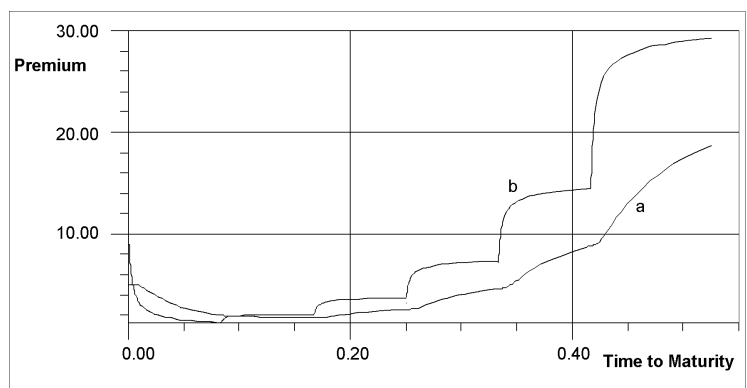

Figure 1: Price of Option as a Function of Time with Underlying at 105 (a) and 109 (b)

\begin{tabular}{|c|c|l|l|}
\hline Underlying & Fair value & Delta & Gamma \\
\hline \hline 80 & 0.18569 & 0.03352 & 0.00493 \\
\hline 90 & 0.91468 & 0.13750 & 0.01774 \\
\hline 100 & 4.48090 & 0.71799 & 0.09950 \\
\hline 105 & 9.35280 & 1.21336 & 0.08385 \\
\hline 109 & 14.63519 & 1.37108 & -0.00449 \\
\hline
\end{tabular}

Table 5: Results Single Barrier Option with Time-dependent Rebate

\subsubsection{Time-dependent Volatilities}

One of the most often criticized weaknesses of the Black-Scholes model is its assumption of constant volatility. This assumption, however, can be dropped without leaving the Black-Scholes environment of lognormal returns. One approach is to assume a term structure of volatility. The most simple model for a term structure of volatility is to assume that the volatility is a linear function of time to maturity:

$$
\sigma(\tau)=a \tau+b
$$

No analytical formula is known for volatility models depending on the moneyness and/or time-to-maturity. This leads to volatility surfaces which are widely used ([51], ch. 14.6). The FEM allows one to integrate complicated deterministic volatility models as shown by [32]. Here we will contrast our results to the results reported by [6] using a trinominal tree. Unfortunately, no details on the trinominal tree calculations are provided. 


\begin{tabular}{|l|c|}
\hline Parameter & Value \\
\hline \hline Asset price & 95 \\
\hline Strike price & 100 \\
\hline Down-and-out barrier & 90 \\
\hline Interest rate & 0.1 \\
\hline Maturity & 1 year \\
\hline
\end{tabular}

Table 6: Data Single Barrier Option with with Time-dependent Volatility

Here we consider constant, increasing, and decreasing volatility:

\begin{tabular}{|c|c|c|c|c|}
\hline Problem & Initial volatility & Ending volatility & $a$ & $b$ \\
\hline \hline 1 & 0.25 & 0.25 & 0 & 0.25 \\
\hline 2 & 0.177 & 0.306 & -0.129 & 0.306 \\
\hline 3 & 0.306 & 0.177 & 0.129 & 0.177 \\
\hline
\end{tabular}

Table 7: Data Volatility Curve

Unfortunately, [6] does not provide any details on his trinominal tree computations.

\begin{tabular}{|c|c|l|l|}
\hline Problem & Method & Fair value & Delta \\
\hline \hline \multirow{3}{*}{1} & Analytical & 5.9968 & 1.120 \\
\cline { 2 - 4 } & FE & 5.9969 & 1.119 \\
\cline { 2 - 4 } & Difference & $0.0017 \%$ & $0.0894 \%$ \\
\hline \hline \multirow{3}{*}{2} & Trinominal tree & 6.4556 & 1.146 \\
\cline { 2 - 4 } & FE & 6.4632 & 1.145 \\
\cline { 2 - 4 } & Difference & $0.1176 \%$ & $0.0873 \%$ \\
\hline \hline \multirow{3}{*}{3} & Trinominal tree & 5.7286 & 1.093 \\
\cline { 2 - 4 } & FE & 5.7169 & 1.089 \\
\cline { 2 - 4 } & Difference & $0.2047 \%$ & $0.3673 \%$ \\
\hline
\end{tabular}

Table 8: Results Volatility Curve 


\subsection{Power Options}

\subsubsection{Plain Vanilla Power Option}

Power options can be subdivided into symmetric and asymmetric power options according to their payoffs:

- symmetric power call: $\max \left((S-X)^{p}, 0\right)$

- asymmetric power call: $\max \left(S^{p}-X, 0\right)$

The payoffs of the puts can be constructed accordingly. To both types, analytical solutions are available ([55], ch. 30). Here, we will contrast our numerical solution to the analytical solutions for the premium, Delta $(\Delta)$, and Gamma $(\Gamma)$. As a basis, we take an example from ([55], p. 589) with the following data:

\begin{tabular}{|l|c|}
\hline Parameter & Value \\
\hline \hline Asset price & 555 \\
\hline Strike price & 550 \\
\hline Interest rate & 0.06 \\
\hline Volatility & 0.15 \\
\hline Dividend Yield & 0.04 \\
\hline Maturity & 0.5 year \\
\hline
\end{tabular}

Table 9: Data Aysmmetric Power Option

In mathematical terms, this can be formulated as following:

$$
\begin{aligned}
\frac{\partial f}{\partial t}+r S \frac{\partial f}{\partial S}+\frac{1}{2} \sigma^{2} S^{2} \frac{\partial^{2} f}{\partial S^{2}} & =r f \\
f(T, S) & =\max \left(S^{p}-X, 0\right) \\
f(t, 0) & =0 \\
f(t, 1000) & =S^{p}-X e^{-r t}
\end{aligned}
$$

Eq. (22) denotes the value of the option deep in the money. It is common practice to cut off the semi-infinite domain at some point to get a finite domain since most numerical routines apply to finite domains ([39], p. 283; [36]; [49]) although numerical techniques for semi-infinite techniques exist ([25]; [53]). The power parameter $p$ is varied. 


\begin{tabular}{|c|c|c|c|c|c|}
\hline$p$ & 0.96 & 0.97 & 0.98 & 0.99 & 1.00 \\
\hline \hline Analytical & 0.17614 & 1.01010 & 4.08800 & 12.21638 & 28.29032 \\
\hline FE & 0.17615 & 1.01080 & 4.08802 & 12.21638 & 28.29040 \\
\hline Difference & $0.0037 \%$ & $0.0027 \%$ & $0.0003 \%$ & $0.0001 \%$ & $0.0003 \%$ \\
\hline \hline$\Delta$ & 0.00892 & 0.04218 & 0.13766 & 0.32420 & 0.58026 \\
\hline$\Delta_{\mathrm{FE}}$ & 0.00892 & 0.04219 & 0.13767 & 0.32421 & 0.58026 \\
\hline Difference & $0.0215 \%$ & $0.0114 \%$ & $0.0064 \%$ & $0.0016 \%$ & $0.0001 \%$ \\
\hline \hline$\Gamma$ & 0.00038 & 0.00141 & 0.00346 & 0.00570 & 0.00648 \\
\hline$\Gamma_{\mathrm{FE}}$ & 0.00039 & 0.00145 & 0.00354 & 0.00576 & 0.00647 \\
\hline Difference & $3.6430 \%$ & $2.7837 \%$ & $2.1628 \%$ & $1.0376 \%$ & $0.0000 \%$ \\
\hline \hline
\end{tabular}

Table 10: Results Asymmetric Power Calls (Part 1)

\begin{tabular}{|c|c|c|c|c|c|}
\hline$p$ & 1.01 & 1.02 & 1.03 & 1.04 & 1.05 \\
\hline \hline Analytical & 53.39500 & 86.29781 & 124.81669 & 167.30009 & 213.01648 \\
\hline FE & 53.39502 & 86.29778 & 124.81670 & 167.30010 & 213.01650 \\
\hline Difference & $0.0000 \%$ & $0.0000 \%$ & $0.0000 \%$ & $0.0000 \%$ & $0.0000 \%$ \\
\hline \hline$\Delta$ & 0.83817 & 1.04341 & 1.19100 & 1.30579 & 1.41124 \\
\hline$\Delta_{\mathrm{FE}}$ & 0.83817 & 1.04340 & 1.19099 & 1.30579 & 1.41124 \\
\hline Difference & $0.0004 \%$ & $0.0011 \%$ & $0.0004 \%$ & $0.0003 \%$ & $0.0001 \%$ \\
\hline \hline$\Gamma$ & 0.00516 & 0.00297 & 0.00129 & 0.00048 & 0.00022 \\
\hline$\Gamma_{\mathrm{FE}}$ & 0.00515 & 0.00291 & 0.00126 & 0.00048 & 0.00022 \\
\hline Difference & $0.3056 \%$ & $2.1197 \%$ & $2.2855 \%$ & $0.2417 \%$ & $1.0919 \%$ \\
\hline
\end{tabular}

Table 11: Results Asymmetric Power Calls (Part 2)

\subsubsection{Capped Power Option}

The Asymmetric Case As mentioned previously, there are closed-form solutions to symmetric and asymmetric power options. But within the market place, only capped power calls and puts with a floor are traded, ${ }^{10}$ for which an analitical solution is not known. In mathematical terms, the problem is to find a solution to the following PDE with the data from the example in sec. 3.2.1:

$$
\begin{aligned}
\frac{\partial f}{\partial t}+r S \frac{\partial f}{\partial S}+\frac{1}{2} \sigma^{2} S^{2} \frac{\partial^{2} f}{\partial S^{2}} & =r f \\
f(T, S) & =\min \left(\max \left(S^{p}-X, 0\right), C\right) \\
f(t, 0) & =0 \\
f(t, 1000) & =50 \\
C & =50
\end{aligned}
$$

\footnotetext{
${ }^{10}$ An Example: In Germany, Bankers Trust has issued capped symmetric FX power options on US \$ (WKN 822512 - WKN 822521), Swiss Francs (WKN 822374, WKN 822376), and Japanese Yen (WKN 826053f) with a power parameter of $p=2$.
} 


\begin{tabular}{|c|c|c|c|c|c|}
\hline$p$ & 0.96 & 0.97 & 0.98 & 0.99 & 1.00 \\
\hline \hline Monte Carlo & 0.163 & 0.909 & 3.442 & 9.327 & 18.887 \\
\hline FE & 0.165 & 1.008 & 3.434 & 9.332 & 18.886 \\
\hline$\Delta_{\mathrm{FE}}$ & 0.00814 & 0.04210 & 0.10882 & 0.21931 & 0.30711 \\
\hline$\Gamma_{\mathrm{FE}}$ & 0.00033 & 0.00141 & 0.00242 & 0.00278 & 0.00097 \\
\hline \hline$p$ & 1.01 & 1.02 & 1.03 & 1.04 & 1.05 \\
\hline \hline Monte Carlo & 29.897 & 39.098 & 44.745 & 47.327 & 48.219 \\
\hline FE & 29.893 & 39.084 & 44.736 & 47.326 & 48.224 \\
\hline$\Delta_{\mathrm{FE}}$ & 0.30619 & 0.22105 & 0.11743 & 0.04658 & 0.01398 \\
\hline$\Gamma_{\mathrm{FE}}$ & -0.00194 & -0.00356 & -0.00304 & -0.00167 & -0.00064 \\
\hline
\end{tabular}

Table 12: Results Capped Asymmetric Power Calls

The Monte Carlo results have been achieved in the most simple way with 1,000,000 samplings.

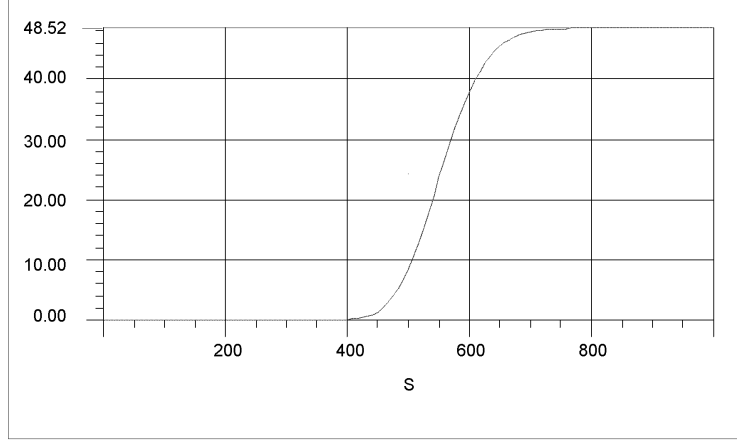

Figure 2: Premium of a Capped Symmetric Power Option

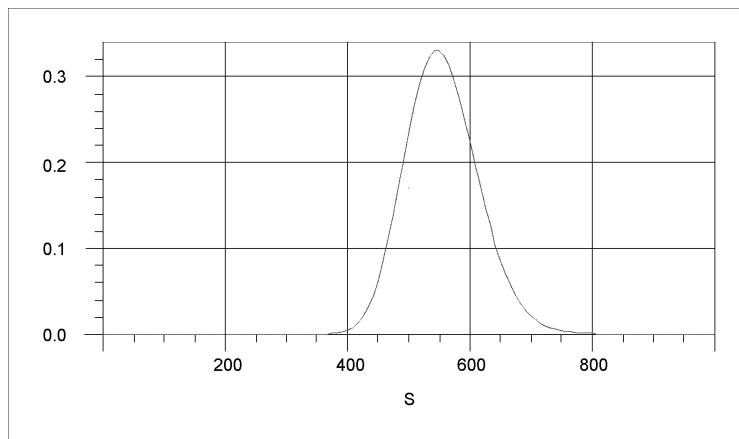

Figure 3: Delta $\Delta$ of a Capped Symmetric Power Option

The Symmetric Case The more popular capped symmetric power option can be formulated accordingly:

$$
\frac{\partial f}{\partial t}+r S \frac{\partial f}{\partial S}+\frac{1}{2} \sigma^{2} S^{2} \frac{\partial^{2} f}{\partial S^{2}}=r f
$$




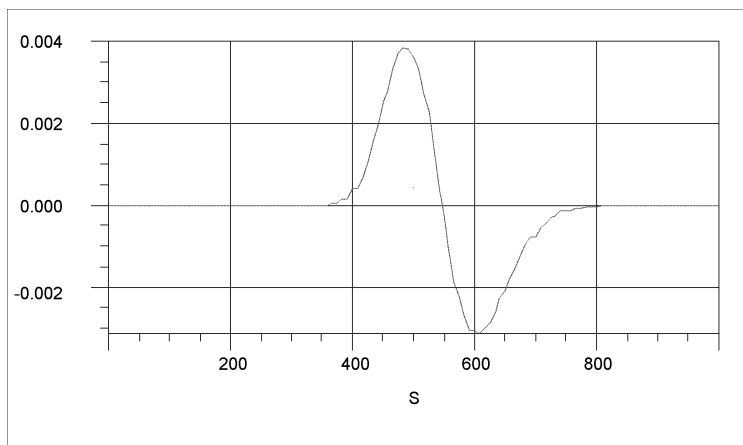

Figure 4: Gamma $\Gamma$ of a Capped Symmetric Power Option

$$
\begin{aligned}
f(T, S) & =\min \left(\max \left((S-X)^{p}, 0\right), C\right) \\
f(t, 0) & =0 \\
f(t, 1000) & =50 \\
C & =50
\end{aligned}
$$

We take the data from table (9) and vary the asset price from out-of-the-money to in-the-money. The power parameter is set to $p=2$ which is predominant in the market place. We compare our results with a simple Monte Carlo approach based on 1,000,000 samplings.

\begin{tabular}{|c|c|c|c|c|c|}
\hline$p$ & 500 & 550 & 555 & 560 & 600 \\
\hline \hline Monte Carlo & 8.47390 & 23.50052 & 25.15097 & 26.78109 & 37.98719 \\
\hline FE & 8.46219 & 23.51419 & 25.16434 & 26.79323 & 37.97783 \\
\hline Difference & $0.1297 \%$ & $0.0582 \%$ & $0.0532 \%$ & $0.1745 \%$ & $0.0246 \%$ \\
\hline$\Delta_{\mathrm{FE}}$ & 0.23545 & 0.33162 & 0.32844 & 0.32311 & 0.22415 \\
\hline$\Gamma_{\mathrm{FE}}$ & 0.00381 & -0.00015 & -0.00064 & -0.00107 & -0.00306 \\
\hline
\end{tabular}

Table 13: Results Capped Symmetric Power Calls

\subsection{Basket Options}

\subsubsection{Put on a Basket}

For options on baskets, at present there is no known analytical solution ([29], p. 161). Therefore, this option has to be priced with a numerical device or an approximation like ([30]; [41]; [55], ch. 27). The basic idea of these approximations is to combine the volatilities of the underlying and their correlations to a single volatility of the basket. This basket is then treated as a single underlying. Using this approach, the problem of pricing an option on a basket is reduced to pricing an option on a single equity. Accordingly, the models to price options with exotic features can also be applied to options on baskets. Precise error estimates are generally not provided ([29], p. 163). Here, however, we price options on baskets using a multi-dimensional PDE. For a plain vanilla put we first derive the boundary conditions. As one or both underlyings become worth much more than the strike, the price of the options goes to zero. As 
the price of first underlying is zero, while the second is positive, the value of the option behaves like the value of a plain vanilla put on a single equity. Therefore, the boundary conditions at $S_{1}=0$ and $S_{2}=0$ are the (time-dependent) solution to the basic Black-Scholes problem of pricing a put ([29], p. 162) with strikes at $\frac{X}{w_{2}}$ and $\frac{X}{w_{1}}$, respectively. Together with the data this becomes the following PDE problem.

\begin{tabular}{|l|c|}
\hline Parameter & Value \\
\hline \hline First asset price & 18 \\
\hline Weight first asset & 1 \\
\hline Second asset price & 20 \\
\hline Weight second asset & 1 \\
\hline Correlation & 0.5 \\
\hline Strike price & 40 \\
\hline Interest rate & 0.1 \\
\hline Dividend Yields & 0.0 \\
\hline
\end{tabular}

Table 14: Data Put on a Basket

$$
\begin{aligned}
\frac{1}{2} \sigma_{1}^{2} S_{1}^{2} \frac{\partial^{2} f}{\partial S_{1}^{2}}+\frac{1}{2} \sigma_{2}^{2} S_{2}^{2} \frac{\partial^{2} f}{\partial S_{2}^{2}} & +\rho \sigma_{1} \sigma_{2} S_{1} S_{2} \frac{\partial^{2} f}{\partial S_{1} \partial S_{2}}+ \\
\left(r-q_{1}\right) S_{1} \frac{\partial f}{\partial S_{1}}+\left(r-q_{2}\right) S_{2} \frac{\partial f}{\partial S_{2}} & =r f-\frac{\partial f}{\partial t} \\
f\left(S_{1}, S_{2}, T\right) & =\max \left(0, X-\left(w_{1} S_{1}+w_{2} S_{2}\right)\right) \text { in } D \\
f\left(S_{1}, 0, t\right) & =g\left(S_{1}, \frac{X}{w_{2}}, t\right) \\
f\left(0, S_{2}, t\right) & =g\left(S_{2}, \frac{X}{w_{1}}, t\right) \\
f\left(100, S_{2}, t\right) & =0 \\
f\left(S_{1}, 100, t\right) & =0
\end{aligned}
$$

Here, the $g$ functions denote a plain vanilla European put with strikes of $\frac{X}{w_{2}}$ and $\frac{X}{w_{1}}$ and appropriate volatilities. We compute the cumulative normal distributions in equations (35) and (36) with an approximation which has four digit accuracy from ([28], p. 243). ${ }^{11}$ To compare the results, we also price the put on a basket using a two-dimension binominal tree as implemented by ([26], ch. 3.3). This tree can be interpreted as a simple explicit finite difference scheme; compare ([50], p. 279).

\footnotetext{
${ }^{11}$ For higher accuracy see also ([28], p. 243f).
} 


\begin{tabular}{|c|c|c|c|c|c|}
\hline \multicolumn{2}{|c|}{ Volatility } & \multicolumn{3}{|c|}{ Time to Maturity } & \multirow[t]{2}{*}{ Premium } \\
\hline$\sigma_{1}^{2}$ & $\sigma_{2}^{2}$ & 0.05 & 0.5 & 0.95 & \\
\hline \multirow{9}{*}{0.1} & \multirow{3}{*}{0.1} & 1.8025 & 0.9543 & 0.6043 & Tree \\
\hline & & 1.8065 & 0.9543 & 0.6035 & FEM \\
\hline & & $0.2204 \%$ & $0.0022 \%$ & $0.1352 \%$ & Diff. \\
\hline & \multirow{3}{*}{0.2} & 1.8333 & 1.4756 & 1.2408 & Tree \\
\hline & & 1.8341 & 1.4764 & 1.2405 & FEM \\
\hline & & $0.0473 \%$ & $0.0512 \%$ & $0.0305 \%$ & Diff. \\
\hline & \multirow{3}{*}{0.3} & 1.9118 & 2.0186 & 1.9265 & Tree \\
\hline & & 1.9138 & 2.0187 & 1.9270 & FEM \\
\hline & & $0.1034 \%$ & $0.0041 \%$ & $0.0242 \%$ & Diff. \\
\hline \multirow{9}{*}{0.2} & \multirow{3}{*}{0.1} & 1.8271 & 1.4120 & 1.1607 & Tree \\
\hline & & 1.8275 & 1.4127 & 1.1601 & FEM \\
\hline & & $0.0236 \%$ & $0.0492 \%$ & $0.0489 \%$ & Diff. \\
\hline & \multirow{3}{*}{0.2} & 1.8859 & 1.8835 & 1.7758 & Tree \\
\hline & & 1.8856 & 1.8833 & 1.7754 & FEM \\
\hline & & $0.0076 \%$ & $0.0125 \%$ & $0.0202 \%$ & Diff. \\
\hline & \multirow{3}{*}{0.3} & 1.9816 & 2.3941 & 2.4389 & Tree \\
\hline & & 1.9830 & 2.3942 & 2.4389 & FEM \\
\hline & & $0.0602 \%$ & $0.0024 \%$ & $0.0004 \%$ & Diff. \\
\hline \multirow{9}{*}{0.3} & \multirow{3}{*}{0.1} & 1.8906 & 1.8941 & 1.7649 & Tree \\
\hline & & 1.8915 & 1.8948 & 1.7647 & FEM \\
\hline & & $0.0451 \%$ & $0.0395 \%$ & $0.0108 \%$ & Diff. \\
\hline & \multirow{3}{*}{0.2} & 1.9683 & 2.3301 & 2.3557 & Tree \\
\hline & & 1.9687 & 2.3298 & 2.3555 & FEM \\
\hline & & $0.0210 \%$ & $0.0138 \%$ & $0.0095 \%$ & Diff. \\
\hline & \multirow{3}{*}{0.3} & 2.0739 & 2.8112 & 2.9985 & Tree \\
\hline & & 2.0747 & 2.8119 & 2.9979 & FEM \\
\hline & & $0.0360 \%$ & $0.0021 \%$ & $0.0181 \%$ & Diff. \\
\hline
\end{tabular}

Table 15: Results Put Option on a Basket Computed on a Square Domain

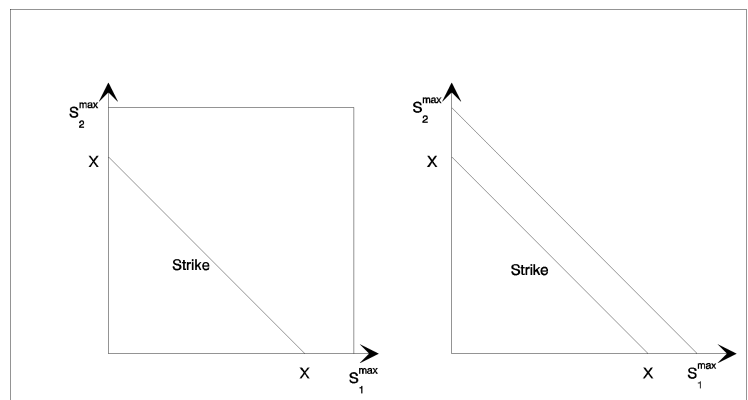

Figure 5: Quadratic and triangular domains for options on baskets

As an alternative to pricing this option on a square domain, we also price it on a triangle, compare fig. (5). Basically, we cut off the section of the domain where the option is totally out of the money and therefore worthless. This, of course, reduces computing time. The boundary conditions (35) to (38) are replaced by:

$$
\begin{aligned}
f\left(S_{1}, 0, t\right) & =g\left(S_{1}, \frac{X}{w_{2}}, t\right) \\
f\left(0, S_{2}, t\right) & =g\left(S_{2}, \frac{X}{w_{1}}, t\right) \\
f\left(S_{1}, S_{2}, t\right) & =0 \text { on } \frac{S_{1}^{\max } S_{2}^{\max }}{}
\end{aligned}
$$




\begin{tabular}{|c|c|c|c|c|c|}
\hline \multicolumn{2}{|c|}{ Volatility } & \multicolumn{3}{|c|}{ Time to Maturity } & \multirow[t]{2}{*}{ Premium } \\
\hline$\sigma_{1}^{2}$ & $\sigma_{2}^{2}$ & 0.05 & 0.5 & 0.95 & \\
\hline \multirow{9}{*}{0.1} & \multirow{3}{*}{0.1} & 1.8026 & 0.9543 & 0.6043 & Tree \\
\hline & & 1.8035 & 0.9545 & 0.6035 & FEM \\
\hline & & $0.0498 \%$ & $0.0155 \%$ & $0.0938 \%$ & Diff. \\
\hline & \multirow{3}{*}{0.2} & 1.8333 & 1.4756 & 1.2408 & Tree \\
\hline & & 1.8334 & 1.4770 & 1.2407 & FEM \\
\hline & & $0.0048 \%$ & $0.0899 \%$ & $0.0097 \%$ & Diff. \\
\hline & \multirow{3}{*}{0.3} & 1.9118 & 2.0186 & 1.9265 & Tree \\
\hline & & 1.9135 & 2.0187 & 1.9262 & FEM \\
\hline & & $0.0919 \%$ & $0.0053 \%$ & $0.0174 \%$ & Diff. \\
\hline \multirow{9}{*}{0.2} & \multirow{3}{*}{0.1} & 1.8271 & 1.4120 & 1.1607 & Tree \\
\hline & & 1.8265 & 1.4119 & 1.1604 & FEM \\
\hline & & $0.0324 \%$ & $0.0095 \%$ & $0.0247 \%$ & Diff. \\
\hline & \multirow{3}{*}{0.2} & 1.8859 & 1.8835 & 1.7758 & Tree \\
\hline & & 1.8850 & 1.8834 & 1.7753 & FEM \\
\hline & & $0.0468 \%$ & $0.0033 \%$ & $0.0256 \%$ & Diff. \\
\hline & \multirow{3}{*}{0.3} & 1.9818 & 2.3941 & 2.4389 & Tree \\
\hline & & 1.9826 & 2.3940 & 2.4387 & FEM \\
\hline & & $0.0426 \%$ & $0.0044 \%$ & $0.0098 \%$ & Diff. \\
\hline \multirow{9}{*}{0.3} & \multirow{3}{*}{0.1} & 1.8906 & 1.8941 & 1.7649 & Tree \\
\hline & & 1.8908 & 1.8937 & 1.7644 & FEM \\
\hline & & $0.0076 \%$ & $0.0218 \%$ & $0.0283 \%$ & Diff. \\
\hline & \multirow{3}{*}{0.2} & 1.9683 & 2.3301 & 2.3557 & Tree \\
\hline & & 1.9679 & 2.3299 & 2.3555 & FEM \\
\hline & & $0.0165 \%$ & $0.0076 \%$ & $0.0081 \%$ & Diff. \\
\hline & \multirow{3}{*}{0.3} & 2.0739 & 2.8120 & 2.9985 & Tree \\
\hline & & 2.0747 & 2.8120 & 2.9988 & FEM \\
\hline & & $0.0368 \%$ & $0.0006 \%$ & $0.0107 \%$ & Diff. \\
\hline
\end{tabular}

Table 16: Results Put Option on a Basket Computed on a Triangular Domain

Although it is possible to adjust FD schemes for non-rectangular domains ([40], ch. 2; [2], p. 258f; [1], sec. 1.9; [10]; [43], sec. 3.4) FE are the natural choice. This is even more true for Neumann conditions which are difficult to integrate into more advanced FD schemes in case of curved boundaries. In a FE setting, however, Neumann conditions are even easier to consider than Dirichlet conditions.

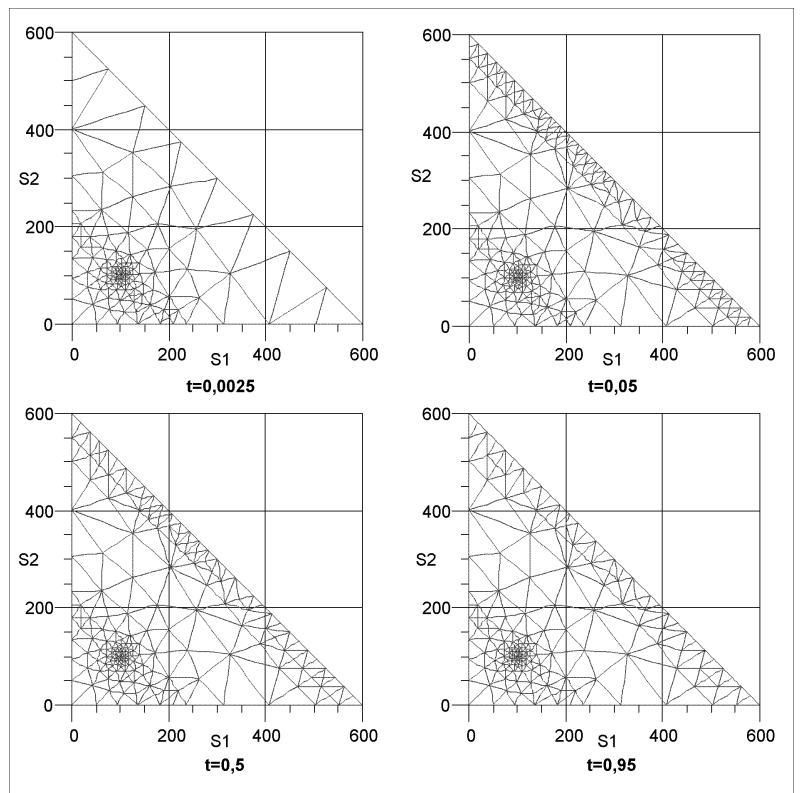

Figure 6: History of FE Mesh

\subsubsection{Call on a Basket}

In [47] Rubinstein reports results on pricing a call on a basket with the following data using a two-dimensional binominal tree: 


\begin{tabular}{|l|c|}
\hline Parameter & Value \\
\hline \hline First asset price & 100 \\
\hline Weight first asset & 1 \\
\hline Second asset price & 100 \\
\hline Weight second asset & 1 \\
\hline Strike price & 200 \\
\hline Correlation & 0.5 \\
\hline Interest rate & 0.0953102 \\
\hline Dividend yield first asset & 0.0487902 \\
\hline Dividend yield second asset & 0.0 \\
\hline
\end{tabular}

Table 17: Data Call on a Basket

With a hundred time steps he achieves the following results:

\begin{tabular}{|c|c|c|r|r|}
\hline \multicolumn{2}{|c|}{ Volatility } & \multicolumn{3}{|c|}{ Time to Maturity } \\
\hline$\sigma_{1}^{2}$ & $\sigma_{2}^{2}$ & 0.05 & 0.5 & 0.95 \\
\hline \hline \multirow{3}{*}{0.1} & 0.1 & 1.92 & 8.97 & 14.70 \\
\cline { 2 - 5 } & 0.2 & 2.72 & 11.22 & 17.45 \\
\cline { 2 - 5 } & 0.3 & 3.58 & 13.70 & 20.59 \\
\hline \multirow{3}{*}{0.2} & 0.1 & 2.72 & 11.15 & 17.28 \\
\cline { 2 - 5 } & 0.2 & 3.45 & 13.33 & 20.13 \\
\cline { 2 - 5 } & 0.3 & 4.24 & 15.72 & 23.25 \\
\hline \multirow{3}{*}{0.3} & 0.1 & 3.57 & 13.56 & 20.25 \\
\cline { 2 - 5 } & 0.2 & 4.24 & 15.65 & 23.08 \\
\cline { 2 - 5 } & 0.3 & 4.99 & 17.94 & 26.16 \\
\hline
\end{tabular}

Table 18: Rubinstein's example with 100 time steps

In order to achieve higher accuracy we redo the example with 200 time steps. These computations have been performed with an implementation of the two-dimension binominal tree by [26]. We compare these results to FE results using the BlackScholes formula for calls on $S_{1}=0$ and $S_{2}=0$ as boundary conditions. We solve the appropriate PDE on a triangular domain assuming a 90 degree outward pointing gradient on the third side. 


\begin{tabular}{|c|c|c|c|c|c|}
\hline \multicolumn{2}{|c|}{ Volatility } & \multicolumn{3}{|c|}{ Time to Maturity } & \multirow{2}{*}{ Method } \\
\hline$\sigma_{1}^{2}$ & $\sigma_{2}^{2}$ & 0.05 & 0.5 & 0.95 & \\
\hline \multirow{9}{*}{0.1} & \multirow{3}{*}{0.1} & 1.9202 & 8.9685 & 14.6980 & Tree \\
\hline & & 1.9198 & 8.9708 & 14.6979 & FEM \\
\hline & & $0.02229 \%$ & $0.02548 \%$ & $0.00055 \%$ & Difference \\
\hline & \multirow{3}{*}{0.2} & 2.7244 & 11.2200 & 17.4460 & Tree \\
\hline & & 2.7316 & 11.2200 & 17.4451 & FEM \\
\hline & & $0.2643 \%$ & $0.0007 \%$ & $0.0050 \%$ & Difference \\
\hline & \multirow{3}{*}{0.3} & 3.5738 & 13.6926 & 20.5925 & Tree \\
\hline & & 3.5805 & 13.6928 & 20.5872 & FEM \\
\hline & & $0.1857 \%$ & $0.0014 \%$ & $0.0258 \%$ & Difference \\
\hline \multirow{9}{*}{0.2} & \multirow{3}{*}{0.1} & 2.7222 & 11.1506 & 17.2815 & Tree \\
\hline & & 2.7317 & 11.1497 & 17.2804 & FEM \\
\hline & & $0.3368 \%$ & $0.0087 \%$ & $0.0061 \%$ & Difference \\
\hline & \multirow{3}{*}{0.2} & 3.4482 & 13.3308 & 20.1281 & Tree \\
\hline & & 3.4563 & 13.3295 & 20.1273 & FEM \\
\hline & & $0.2357 \%$ & $0.0093 \%$ & $0.0038 \%$ & Difference \\
\hline & \multirow{3}{*}{0.3} & 4.2420 & 15.7150 & 23.2494 & Tree \\
\hline & & 4.2473 & 15.7138 & 23.2472 & FEM \\
\hline & & $0.1252 \%$ & $0.0079 \%$ & $0.0097 \%$ & Difference \\
\hline \multirow{9}{*}{0.3} & \multirow{3}{*}{0.1} & 3.5689 & 13.5534 & 20.2429 & Tree \\
\hline & & 3.5779 & 13.5501 & 20.2406 & FEM \\
\hline & & $0.2530 \%$ & $0.0244 \%$ & $0.0110 \%$ & Difference \\
\hline & \multirow{3}{*}{0.2} & 4.2398 & 15.6421 & 23.0708 & Tree \\
\hline & & 4.2453 & 15.6408 & 23.0686 & FEM \\
\hline & & $0.1310 \%$ & $0.0090 \%$ & $0.0095 \%$ & Difference \\
\hline & \multirow{3}{*}{0.3} & 4.9846 & 17.9382 & 26.1506 & Tree \\
\hline & & 4.9878 & 17.9362 & 26.1459 & FEM \\
\hline & & $0.0640 \%$ & $0.0110 \%$ & $0.01798 \%$ & Difference \\
\hline
\end{tabular}

Table 19: Rubinstein's example with Dirichlet Boundary Conditions

Redoing the above example assuming a zero gradient on $S_{1}=0$ and $S_{2}=0$ leads to slightly less accurate results. This Neumann condition is obviously the easiest to apply.

\begin{tabular}{|c|c|c|c|c|c|}
\hline \multicolumn{2}{|c|}{ Volatility } & \multicolumn{3}{|c|}{ Time to Maturity } & \multirow[t]{2}{*}{ Method } \\
\hline$\sigma_{1}^{2}$ & $\sigma_{2}^{2}$ & 0.05 & 0.5 & 0.95 & \\
\hline \multirow{9}{*}{0.1} & \multirow{3}{*}{0.1} & 1.9202 & 8.9685 & 14.6980 & Tree \\
\hline & & 1.9198 & 8.9712 & 14.6982 & FEM \\
\hline & & $0.0223 \%$ & $0.0303 \%$ & $0.0020 \%$ & Difference \\
\hline & \multirow{3}{*}{0.2} & 2.7244 & 11.2199 & 17.4460 & Tree \\
\hline & & 2.7316 & 11.2210 & 17.4461 & FEM \\
\hline & & $0.2644 \%$ & $0.0097 \%$ & $0.0004 \%$ & Difference \\
\hline & \multirow{3}{*}{0.3} & 3.5738 & 13.6926 & 20.5925 & Tree \\
\hline & & 3.5805 & 13.6942 & 20.5889 & FEM \\
\hline & & $0.1855 \%$ & $0.0112 \%$ & $0.0177 \%$ & Difference \\
\hline \multirow{9}{*}{0.2} & \multirow{3}{*}{0.1} & 2.7222 & 11.1507 & 17.2815 & Tree \\
\hline & & 2.7314 & 11.1506 & 17.2814 & FEM \\
\hline & & $0.3368 \%$ & $0.0005 \%$ & $0.0006 \%$ & Difference \\
\hline & \multirow{3}{*}{0.2} & 3.4482 & 13.3308 & 20.1281 & Tree \\
\hline & & 3.4563 & 13.3306 & 20.1286 & FEM \\
\hline & & $0.2358 \%$ & $0.0013 \%$ & $0.0028 \%$ & Difference \\
\hline & \multirow{3}{*}{0.3} & 4.2420 & 15.7150 & 23.2494 & Tree \\
\hline & & 4.2473 & 15.7152 & 23.2488 & FEM \\
\hline & & $0.1253 \%$ & $0.0016 \%$ & $0.0024 \%$ & Difference \\
\hline \multirow{9}{*}{0.3} & \multirow{3}{*}{0.1} & 3.5689 & 13.5534 & 20.2429 & Tree \\
\hline & & 3.5779 & 13.5517 & 20.2421 & FEM \\
\hline & & $0.2530 \%$ & $0.0129 \%$ & $0.0035 \%$ & Difference \\
\hline & \multirow{3}{*}{0.2} & 4.2398 & 15.6421 & 23.0708 & Tree \\
\hline & & 4.2454 & 15.6420 & 23.0704 & FEM \\
\hline & & $0.1313 \%$ & $0.0006 \%$ & $0.0017 \%$ & Difference \\
\hline & \multirow{3}{*}{0.3} & 4.9846 & 17.9382 & 26.1506 & Tree \\
\hline & & 4.9878 & 17.9376 & 26.1477 & FEM \\
\hline & & $0.0640 \%$ & $0.0031 \%$ & $0.0109 \%$ & Difference \\
\hline
\end{tabular}

Table 20: Rubinstein's example with Neumann Boundary Conditions

\subsubsection{Single Barrier Knock-Out Call on a Basket}

\section{Without Rebate}

For the knock-out call on a basket the boundaries for $S_{1}=0$ and $S_{2}=0$ first have to be computed numerically due to the reasons explained in chapter 3.1. The third 


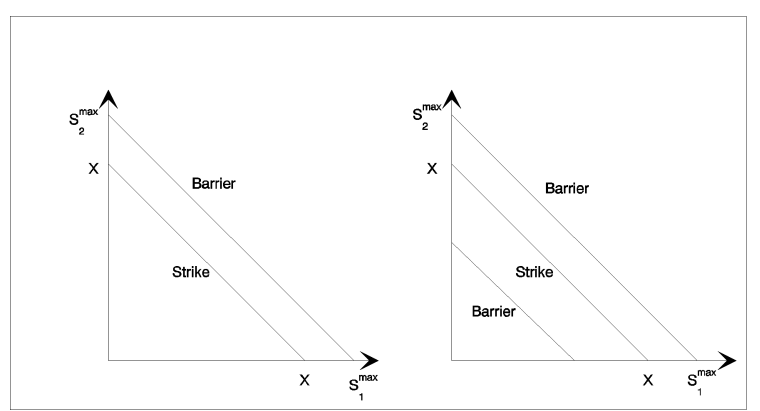

Figure 7: Domains of a Single and Double Barrier Knock-out Call

boundary is the rebate $R=10$. This leads to the following system of PDEs. Eq. (42) to (45) denote a barrier call on $S_{1}$ with $S_{2}=0$. Accordingly, eq. (46) to (49) denote a barrier call on $S_{2}$ with $S_{1}=0$

$$
\begin{aligned}
\frac{\partial f_{1}}{\partial t}+r S \frac{\partial f_{1}}{\partial S_{1}}+\frac{1}{2} \sigma_{1}^{2} S_{1}^{2} \frac{\partial^{2} f_{1}}{\partial S_{1}^{2}} & =r f_{1} \\
f\left(T, S_{1}\right) & =\max \left(S_{1}-X, 0\right) \\
f(t, 0) & =0 \\
f\left(t, S_{1}^{\max }\right) & =R \\
\frac{\partial f_{2}}{\partial t}+r S \frac{\partial f_{2}}{\partial S_{2}}+\frac{1}{2} \sigma_{2}^{2} S_{2}^{2} \frac{\partial^{2} f_{2}}{\partial S_{2}^{2}} & =r f_{2} \\
f\left(T, S_{2}\right) & =\max \left(S_{2}-X, 0\right) \\
f(t, 0) & =0 \\
f\left(t, S_{2}^{\max }\right) & =R \\
\frac{1}{2} \sigma_{2}^{2} S_{2}^{2} \frac{\partial^{2} f_{3}}{\partial S_{2}^{2}} & +\rho \sigma_{1} \sigma_{2} S_{1} S_{2} \frac{\partial^{2} f_{3}}{\partial S_{1} \partial S_{2}}+ \\
\left(r-q_{1}\right) S_{1}^{2} \frac{\partial^{2} f_{3}}{\partial S_{1}^{2}} \frac{\partial f_{3}}{\partial S_{1}}+\left(r-q_{2}\right) S_{2} \frac{\partial f_{3}}{\partial S_{2}} & =r f_{3}-\frac{\partial f_{3}}{\partial t} \\
f_{3}\left(S_{1}, S_{2}, T\right) & =\max \left(0, X-\left(w_{1} S_{1}+w_{2} S_{2}\right)\right) \text { in } D \\
f_{3}\left(S_{1}, 0, t\right) & =f_{1}\left(S_{1}, t\right) \\
f_{3}\left(0, S_{2}, t\right) & =f_{2}\left(S_{2}, t\right) \\
f_{3}\left(S_{1}, S_{2}, t\right) & =R \text { on } \overline{S_{1}^{\max } S_{2}^{\max }}
\end{aligned}
$$

The parameters are again taken from Rubinstein's example. We solve this problem as a system although it could be solved sequentially. Solving this problem as system avoids having to feed the numerical solutions back into the program. We do not have a direct way of checking the results but the premiums should be below the ones from Rubinstein's example due to the knock-out feature (which they are). 


\begin{tabular}{|c|c|c|c|c|}
\hline \multicolumn{2}{|c|}{ Volatility } & \multicolumn{3}{|c|}{ Time to Maturity } \\
\hline$\sigma_{1}^{2}$ & $\sigma_{2}^{2}$ & 0.05 & 0.5 & 0.95 \\
\hline \hline \multirow{3}{*}{0.1} & 0.1 & 1.7416 & 0.4645 & 0.1771 \\
\cline { 2 - 5 } & 0.2 & 1.5198 & 0.1532 & 0.0581 \\
\cline { 2 - 5 } & 0.3 & 1.0738 & 0.0643 & 0.0246 \\
\hline \multirow{3}{*}{0.2} & 0.1 & 1.5218 & 0.1568 & 0.0608 \\
\cline { 2 - 5 } & 0.2 & 1.1074 & 0.0727 & 0.0273 \\
\cline { 2 - 5 } & 0.3 & 0.7361 & 0.0375 & 0.0140 \\
\hline \multirow{3}{*}{0.3} & 0.1 & 1.0199 & 0.0665 & 0.0264 \\
\cline { 2 - 5 } & 0.2 & 0.7368 & 0.0381 & 0.0144 \\
\cline { 2 - 5 } & 0.3 & 0.5108 & 0.0225 & 0.0083 \\
\hline
\end{tabular}

Table 21: Results Knock-out Call on a Basket without Rebate

With Rebate By introducing a rebate of $R=10$ the problem above looses a lot of its curvature. Again, we do not have a direct way of checking the results. By arbitrage considerations, however, each premium should be worth more than without rebate and less than in Rubinstein's example. This is satisfied as can be checked easily by inspecting the tables (21) and (19).

\begin{tabular}{|c|c|c|c|c|}
\hline \multicolumn{2}{|c|}{ Volatility } & \multicolumn{3}{|c|}{ Time to Maturity } \\
\hline$\sigma_{1}^{2}$ & $\sigma_{2}^{2}$ & 0.05 & 0.5 & 0.95 \\
\hline \hline \multirow{3}{*}{0.1} & 0.1 & 1.9161 & 6.5008 & 7.8353 \\
\cline { 2 - 5 } & 0.2 & 2.6963 & 7.0624 & 8.0269 \\
\cline { 2 - 5 } & 0.3 & 3.4802 & 7.5058 & 8.2390 \\
\hline \multirow{3}{*}{0.2} & 0.1 & 2.6944 & 7.0598 & 8.0308 \\
\cline { 2 - 5 } & 0.2 & 3.3708 & 7.4913 & 8.2597 \\
\cline { 2 - 5 } & 0.3 & 4.0531 & 7.8185 & 8.4395 \\
\hline \multirow{3}{*}{0.3} & 0.1 & 3.4771 & 7.5017 & 8.2416 \\
\cline { 2 - 5 } & 0.2 & 4.0517 & 7.8177 & 8.4410 \\
\cline { 2 - 5 } & 0.3 & 4.6210 & 8.0668 & 8.5942 \\
\hline
\end{tabular}

Table 22: Results Knock-out Call on a Basket with Rebate

\subsubsection{Double Barrier Knock-Out Call on a Basket}

In addition to the example above, in table (24) we introduce a second down-and-out barrier at a value of the basket of 100 . No rebate is paid on this barrier. The domain now turns into an irregular strip. 


\begin{tabular}{|c|c|c|c|c|}
\hline \multicolumn{2}{|c|}{ Volatility } & \multicolumn{3}{|c|}{ Time to Maturity } \\
\hline$\sigma_{1}^{2}$ & $\sigma_{2}^{2}$ & 0.05 & 0.5 & 0.95 \\
\hline \hline \multirow{3}{*}{0.1} & 0.1 & 1.9272 & 6.4709 & 7.8227 \\
\cline { 2 - 5 } & 0.2 & 2.6108 & 7.0515 & 8.0222 \\
\cline { 2 - 5 } & 0.3 & 3.4162 & 7.5003 & 8.2368 \\
\hline \multirow{3}{*}{0.2} & 0.1 & 2.6218 & 7.0465 & 8.0245 \\
\cline { 2 - 5 } & 0.2 & 3.3089 & 7.4847 & 8.2566 \\
\cline { 2 - 5 } & 0.3 & 4.0078 & 7.8149 & 8.4379 \\
\hline \multirow{3}{*}{0.3} & 0.1 & 3.4221 & 7.4949 & 8.2383 \\
\cline { 2 - 5 } & 0.2 & 4.0064 & 7.8139 & 8.4394 \\
\cline { 2 - 5 } & 0.3 & 4.5877 & 8.0653 & 8.5907 \\
\hline & \multicolumn{4}{|c}{} \\
\hline
\end{tabular}

Table 23: Results Double Barrier Knock-out Call on a Basket

\begin{tabular}{|c|c|c|c|c|}
\hline \multicolumn{2}{|c|}{ Volatility } & \multicolumn{3}{|c|}{ Time to Maturity } \\
\hline$\sigma_{1}^{2}$ & $\sigma_{2}^{2}$ & 0.05 & 0.5 & 0.95 \\
\hline \hline \multirow{3}{*}{0.1} & 0.1 & 1.9272 & 6.4709 & 7.8227 \\
\cline { 2 - 5 } & 0.2 & 2.6108 & 7.0515 & 8.0222 \\
\cline { 2 - 5 } & 0.3 & 3.4162 & 7.5003 & 8.2368 \\
\hline \multirow{3}{*}{0.2} & 0.1 & 2.6218 & 7.0465 & 8.0245 \\
\cline { 2 - 5 } & 0.2 & 3.3089 & 7.4847 & 8.2566 \\
\cline { 2 - 5 } & 0.3 & 4.0078 & 7.8149 & 8.4379 \\
\hline \multirow{3}{*}{0.3} & 0.1 & 3.4221 & 7.4949 & 8.2383 \\
\cline { 2 - 5 } & 0.2 & 4.0064 & 7.8139 & 8.4394 \\
\cline { 2 - 5 } & 0.3 & 4.5877 & 8.0653 & 8.5907 \\
\hline
\end{tabular}

Table 24: Results Knock-out Call on a Basket with Rebate

The plausibility of these results can be checked with table (24). They are slightly lower than in the example without down-and-out barrier. Since this additional barrier is deeply out of the money it does have only little impact.

\subsubsection{Capped Call on a Basket}

Analytical Boundary Conditions The pricing of this product ${ }^{12}$ leads to the PDE eq. (34) with the following initial and boundary conditions:

$$
\begin{aligned}
f\left(S_{1}, S_{2}, 0\right) & =\min \left(\operatorname{cap}, \max \left(0, X-\left(w_{1} S_{1}+w_{2} S_{2}\right)\right)\right. \\
f\left(S_{1}, 0, t\right) & =g\left(S_{1}, \frac{X}{w_{2}}, t\right)-g\left(S_{1}, \text { cap }, t\right) \\
f\left(0, S_{2}, t\right) & =g\left(S_{2}, \frac{X}{w_{1}}, t\right)-g\left(S_{2}, \text { cap }, t\right) \\
f\left(S_{1}, S_{2}, t\right) & =0 \text { on } \frac{S_{1}^{\max } S_{2}^{\max }}{}
\end{aligned}
$$

\footnotetext{
${ }^{12}$ In Germany, examples for capped basket options are WKN 822361, WKN 822362, WKN 822380, and WKN 822399 which are traded at the stock exchanges in Frankfurt, Düsseldorf, and Stuttgart.
} 
The data, again, are taken from Rubinstein's example with an additional cap of 10. Again, this PDE can be solved either on a square or triangular domain. For reasons outlined above, we chose the triangle. The boundary conditions at $S_{1}=0$ and $S_{2}=0$ represent the prices of capped European call options with strike prices of $\frac{X}{w_{2}}$ and $\frac{X}{w_{1}}$, respectively. This capped call can be priced either by entering a bull spread and pricing its parts individually with the analytical formula for European calls or numerically.

\begin{tabular}{|c|c|c|c|c|}
\hline \multicolumn{2}{|c|}{ Volatility } & \multicolumn{3}{|c|}{ Time to Maturity } \\
\hline$\sigma_{1}^{2}$ & $\sigma_{2}^{2}$ & 0.05 & 0.5 & 0.95 \\
\hline \hline \multirow{3}{*}{0.1} & 0.1 & 1.9062 & 5.3075 & 6.1865 \\
\cline { 2 - 5 } & 0.2 & 2.5549 & 4.9940 & 5.4814 \\
\cline { 2 - 5 } & 0.3 & 3.0139 & 4.7564 & 5.0111 \\
\hline \multirow{3}{*}{0.2} & 0.1 & 2.5534 & 5.0002 & 5.5096 \\
\cline { 2 - 5 } & 0.2 & 2.9701 & 4.8713 & 5.1819 \\
\cline { 2 - 5 } & 0.3 & 3.2831 & 4.7214 & 4.8849 \\
\hline \multirow{3}{*}{0.3} & 0.1 & 3.0124 & 4.7643 & 5.0459 \\
\cline { 2 - 5 } & 0.2 & 3.2825 & 4.7260 & 4.9005 \\
\cline { 2 - 5 } & 0.3 & 3.4980 & 4.6436 & 4.7225 \\
\hline
\end{tabular}

Table 25: Results Capped Call on a Basket with Analytical Boundary Conditions

Again, we do not have a direct way of checking the results. By arbitrage considerations, however, each premium should be worth less than the example above with a rebate of $R=10$ table (24) and more than the example without rebate table $(21)$.

Gradient Boundary Conditions An alternative to eq. (56 and (57) is to assume a zero gradient on $S_{1}=0$ and $S_{2}=0$. The results differ only slightly; compare table (26).

\begin{tabular}{|c|c|c|c|c|}
\hline \multicolumn{2}{|c|}{ Volatility } & \multicolumn{3}{|c|}{ Time to Maturity } \\
\hline$\sigma_{1}^{2}$ & $\sigma_{2}^{2}$ & 0.05 & 0.5 & 0.95 \\
\hline \hline \multirow{3}{*}{0.1} & 0.1 & 1.9073 & 5.3112 & 6.1885 \\
\cline { 2 - 5 } & 0.2 & 2.5604 & 4.9959 & 5.4823 \\
\cline { 2 - 5 } & 0.3 & 3.0206 & 4.7575 & 5.0116 \\
\hline \multirow{3}{*}{0.2} & 0.1 & 2.5559 & 5.0002 & 5.5110 \\
\cline { 2 - 5 } & 0.2 & 2.9735 & 4.8723 & 5.1825 \\
\cline { 2 - 5 } & 0.3 & 3.2873 & 4.7220 & 4.8853 \\
\hline \multirow{3}{*}{0.3} & 0.1 & 3.0138 & 4.7654 & 5.0467 \\
\cline { 2 - 5 } & 0.2 & 3.2844 & 4.7266 & 4.9011 \\
\cline { 2 - 5 } & 0.3 & 3.5009 & 4.6442 & 4.7223 \\
\hline
\end{tabular}

Table 26: Results Capped Call on a Basket with Numerical Boundary Conditions

\subsubsection{Capped Power Call on a Basket with a Down-and-out Barrier}

In this section we apply some of the exotic features of the previous sections on a symmetric power call on a basket. The power parameter is set to $p=2$. Additionally, 
we introduce a cap of 20 on the option and a down-and-out barrier when the basket becomes worth less than 100 .

\begin{tabular}{|c|c|c|c|c|}
\hline \multicolumn{2}{|c|}{ Volatility } & \multicolumn{3}{|c|}{ Time to Maturity } \\
\hline$\sigma_{1}^{2}$ & $\sigma_{2}^{2}$ & 0.05 & 0.5 & 0.95 \\
\hline \hline \multirow{3}{*}{0.1} & 0.1 & 5.5636 & 11.8290 & 13.1566 \\
\cline { 2 - 5 } & 0.2 & 6.8347 & 10.7868 & 11.5071 \\
\cline { 2 - 5 } & 0.3 & 7.5183 & 10.1014 & 10.4334 \\
\hline \multirow{3}{*}{0.2} & 0.1 & 6.8742 & 10.8144 & 11.5788 \\
\cline { 2 - 5 } & 0.2 & 7.5100 & 10.3587 & 10.7864 \\
\cline { 2 - 5 } & 0.3 & 7.9196 & 9.9305 & 10.1068 \\
\hline \multirow{3}{*}{0.3} & 0.1 & 7.5528 & 10.1310 & 10.5182 \\
\cline { 2 - 5 } & 0.2 & 7.9261 & 9.9433 & 10.1431 \\
\cline { 2 - 5 } & 0.3 & 8.1958 & 9.6955 & 9.7287 \\
\hline
\end{tabular}

Table 27: Results Power Call on a Basket with Floor and Knock-out Barrier

\section{Conclusions}

In the previous sections it has been demonstrated how to use FE to price options of various kinds. It has been delineated that the FEM has some advantages in computing accurate Greeks due to its polynominal approximation of the PDE. It has also been outlined how non-rectangular domains arise in option pricing and how to deal with these in a FE setting. This has been demonstrated with various options on baskets, but this can easily be generalized to other rainbow options. The possibility of being able to handle arbitrary domains is the main reason for the predominance of FE in civil and mechanical engineering. This allows a wealth of knowledge and software to be on hand. The package used for this paper is $P D E$ ase $2 D^{T M}$, clearly its high accuracy has been demonstrated. A computer run for a single problem takes from a few seconds to several minutes. ${ }^{13}$ Since $P D E$ ase $2 D^{T M}$ is a general purpose program for linear and nonlinear PDEs of various types and arbitrary domains, the solution process could be made substantially faster by coding only parabolic PDEs.

\footnotetext{
${ }^{13}$ Since many different PCs were used for this paper, CPU time of individual problems are not shown.
} 


\section{References}

[1] Ames, W. F.: Numerical Methods for Partial Differential Equations, 3. ed.. Boston etc., 1992.

[2] Bellomo, N., Preziosi, L.: Modelling Mathematical Methods and Scientific Computing. Boca Raton (Florida), 1995.

[3] Berger, E.: Barrier Options in: [44]

[4] Bickford, W. B.: A First Course in the Finite Element Method. Boston, 1990.

[5] Black, F., Scholes, M.: The Pricing of Options and Corporate Liabilities. Journal of Political Economy, 81 (1973) 637-659.

[6] Brenner, R. J.: Volatility is not Constant. in: [44].

[7] Broadie, M., Glassermann, P.: A Continuity Correction for Discrete Barrier Options. Mathematical Finance, 7 (1997) 325-349.

[8] Burnett, D. S.: Finite Element Analysis - From Concepts to Applications. Reading (US) etc., 1987.

[9] Chriss, N., Tsiveriotis, K.: Pricing with a Difference. Risk, February 1998, 8083.

[10] Collatz, L: The Numerical Treatment of Differential Equations. Berlin etc., 1966.

[11] Crank, J.: Free and Moving Boundary Problems. Oxford, 1984.

[12] Dempster, M. A. H., Hutton, J. P.: Numerical Valuation of Cross-Currency Swaps and Swaptions. Version October 1996. Discussion Paper. Department of Mathematics. University of Essex.

[13] Dempster, M. A. H., Hutton, J. P.: Numerical Valuation of Cross-Currency Swaps and Swaptions in: Dempster, M. A. H., Pliska, St. R. (eds.): Mathematics of Derivative Securities. Cambridge (UK), 1997.

[14] Dixit, A. K., Pindyck, R. S.: Investment under Uncertainty. Princeton (USA), 1994.

[15] Druskin, V., Knizhnerman, Tamarchenko, T., Kostek, S.: Krylov Subspace Reduction and its Extensions for Option Pricing. Journal of Computational Finance, 1 (1997) 63-79.

[16] Duffie, D.: The Theory of Value in Security Markets in: Hildenbrand, W., Sonnenschein, H.: Handbook of Mathematical Economics Vol. IV. NorthHolland etc., 1991.

[17] Duffie, D.: Dynamic Asset Pricing Theory, 2. ed.. Princeton (USA), 1996. 
[18] Duffie, D., Kan, R.: A Yield-Factor Model of Interest Rates. Mathematical Finance. 6 (1996) 379-406.

[19] Eriksson K., Estep, D., Hansbo, P., Johnson C: Computational Differential Equations. Lund (Sweden), 1996.

[20] Faires, J. D., Burden, R.: Numerical Methods, 2. ed. Pacific Grove etc., 1998.

[21] Forsyth, P. A., Vetzal, K. R., Zvan, R.: A Finite Element Approach to the Pricing of Discrete Lookbacks with Stochastic Volatility. Preprint University of Waterloo, Department of Computer Science. Version July 11, 1997.

[22] Forsyth, P. A., Vetzal, K. R., Zvan, R.: Penalty Methods for American Options with Stochastic Volatility. Preprint University of Waterloo, Department of Computer Science. Version September 30, 1997.

[23] Forsyth, P. A., Vetzal, K. R., Zvan, R.: A General Finite Element Approach for PDE Option Pricing Models. Preprint University of Waterloo. Version December 1998.

[24] Geske, R., Shastri, K.: Valuation by Approximation: A Comparison of Alternative Option Valuation Techniques. Journal of Finance and Quantitative Analysis, 20/1 1985 45-71.

[25] Givoli, D.: Numerical Methods for Problems in Infinite Domains. Amsterdam etc., 1992.

[26] Haug, E. G.: The Complete Guide to Option Pricing Formulas. New York, 1997.

[27] Hinton, E., Campbell, J. S.: Local and Global Smoothing of Discontinuous Finite Element Functions Using a Least Square Method. International Journal for Numerical Methods in Engineering. 8 (1974) 461-480.

[28] Hull, J.: Options, Futures and Other Derivatives, 3. ed.. London etc., 1997.

[29] Hunziker, J. P., Koch-Medina P.: Two-Color Rainbow Options in: [44].

[30] Huynh, Ch. B.: Back to Baskets. Risk. 7/5 (1994).

[31] Jackson, N., Süli, E.: Adaptive Finite Element Solution of 1D European Option Pricing Problems. Oxford University Computing Laboratory. Report 97/05.

[32] Jackson, N., Süli, E., Howison, S.: Computation of Deterministic Volatility Surfaces. Oxford University Computing Laboratory. Report 98/01.

[33] Jarrow, R., Turnbull, S.: Derivative Securities. Cincinatti, 1996.

[34] Johnson, C.: Numerical Solution of Partial Differential Equations by the Finite Element Method. 3. ed.. New York etc., 1990.

[35] Kunitomo, N., Ikeda, M.: Pricing Options with Curved Boundaries. Mathematical Finance, 2/4 (1992) 272-298. 
[36] Kushner, H. J.: Numerical Methods for Stochastic Control Problems in Finance in: Dempster, M. A. H., Pliska, St. R. (eds.): Mathematics of Derivative Securities. Cambridge (UK), 1997.

[37] Leland, H. E.: Option Pricing and Replication with Transaction Costs. Journal of Finance, 40 (1985) 1283-1301.

[38] Macsyma Inc. (ed.): PDEase2D ${ }^{T M}$ Reference Manual, 3. ed.. Arlington (USA), 1996.

[39] McIver, J. L.: Overview of Modeling Techniques in: [44].

[40] Mitchell, A. R., Griffiths, D. F.: The Finite Difference Method in Partial Differential Equations. Chichester etc., 1980.

[41] Milevsky, M. A., Posner, St. E.: A Closed-form Approximation for Valuing Basket Options. Journal of Derivatives. (1998) 54-61.

[42] Moan, T.: On the Local Distribution of Errors by Finite Element Approximations. in: Yamada, Y., Gallagher (eds): Theory and Practice in Finite Element Structural Analysis. Tokyo, 1973.

[43] Morton, K. W., Mayer, D. F.: Numerical Solution of Partial Differential Equations. Cambridge (UK), 1994.

[44] Nelken, Israel: The Handbook of Exotic Options. Chicago etc., 1996.

[45] Quateroni, A., Valli, A.: Numerical Approximation of Partial Differential Equations. Berlin etc., 1994.

[46] Rebonato, R.: Interest-Rate Option Models: A Critical Survey in: Alexander, C.: The Handbook of Risk Management and Analysis, Chichester, 1997.

[47] Rubinstein, M.: Somewhere over the Rainbow. Risk. 11/2 (1991).

[48] Topper, J.: Solving Term Structure Models with Finite Elements. Marburg, 1998.

[49] Topper, J.: Some Remarks on the Approximation of Boundaries in PDE-based Financial Models. Discussion Paper, Department of Economics, University of Hannover, to appear.

[50] Wilmott, P., Dewynne, J., Howison, S.: Option Pricing - Mathematical Models and Computation. Oxford, 1996.

[51] Wilmott, P.: Derivatives. Chichester etc., 1998.

[52] White, R. E.: An Introduction to the Finite Element Method with Applications to Nonlinear Problems. New York etc., 1985.

[53] Wolf, J. P., Song, Ch.: Finite Element Modelling of Unbounded Media. Chichester etc., 1996. 
[54] Ženíšek, A.: Nonlinear Elliptic and Evolution Problems and their Finite Element Approximation. London etc., 1990.

[55] Zhang, P. G.: Exotic Options. Singapore etc., 1997. 